Ann. Zootech., I963, 12 (3), I8I-Ig2.

\title{
ECTROMÉLIE ET OTOCÉPHALIE HÉRÉDITAIRES EN RACE FRANÇAISE FRISONNE PIE NOIRE
}

\author{
J. J. LAUVERGNE et I'. CUQ \\ Station centrale de Génétique animale, \\ Centre national de Recherches zootechniques, Jouy-en-Josas (Seine-el-Oise); \\ Laboratoire d'Anatomie, Ecole nationale vétérinaire, Alfort (Seine)
}

SOMMAIRE

Une anomalie est apparue à plusieurs reprises, en race Française Frisonne Pie Noire, dans la zone d'un Centre d'Insémination du Nord de la France.

Chez tous les sujets atteints l'absence plus ou moins complète des membres (ectromélie) s'accom. pagne de malformations de la tête (otocéphalie). Les anormaux naissent mort-nés ì la suite d'une durée de gestation plus courte que la normale.

L'hypothèse génétique la plus vraisemblable -- admise d'ailleurs dans la littérature - est que ce caractère est conditionné par un facteur autosomal récessif.

Les pères des monstres que nous avons étudiés proviennent de Frise et ils sont apparentés à un taureau qui, aux Pays-Bas, a donné des veaux " amputés». Il y a donc lieu de penser que l'anomalie évoquée est une réapparition de l'ectromélie récessive associée à l'otocéphalie, latente en race Pie Noire Frisonne : " acroteriasis congenita » de WrIEDT et MoHr.

\section{INTRODUC'TION}

L'anomalie étudiée dans ce mémoire a été signalée au début de l'année I96I en race Française Frisonne Pie Noire dans le cadre de la cone d'action d'un Centre d'Insémination Artificielle du Nord de la France.

Il s'agissait de monstres non viables dont les caractéristiques principales étaient la réduction des membres à l'état de moignon et l'absence plus ou moins complète de mâchoire inférieure.

Dès 1'apparition des premiers monstres, la direction du Centre a entrepris une enquête sur les descendants de ses taureaux Firisons à naître entre septembre I96r et février Ig62. 
Cette enquête a révélé l'existence de 26 anormaux dont la description extérieure et la généalogie ont pu être données dans un certain nombre de cas.

Un de ces animaux a été disséqué au laboratoire de la Chaire d'Anatomie de l'École vétérinaire d'Alfort, ce qui a permis de préciser la base anatomique des monstruosités observées.

Les liaisons de parenté entre les pères et grand-pères des anormaux ont été établies d'après les pedigrees đu Herd-Book de la race française Frisonne Pie Noire (HBFFPN) et du Herd-Book Frison [Friesch Rundvee -Stamboek (FRS)] de Leeuwarden (Pays-Bas).

I.es informations en provenance de ces trois sources (enquête, dissection, pedigrees) seront présentées dans l'ordre suivant:

- Sexe, viabilité, durée de gestation des anormaux.

- Description morphologique.

- Données de parenté des anormaux.

\section{RÉSULTATS}

\section{I. - Sexe, VIAbirit'F́, DURÉE DË Gestation}

Des 26 monstres recueillis lors de l'enquête, to étaient de sexe mâle, deux de sexe femelle et I4 de sexe non précisé.

Tous les anormaux étaient mort-nés ou mouraient peu après la naissance et dans deux cas au moins la mort était intervenue longtemps avant la mise bas car le fotus était décomposé.

Les durées de gestation étaient connues avec précision dans $r_{5}$ cas $\left(70^{\pi} 0^{\pi}, 2 \%\right.$ six animaux de sexe non précisé). Elles s'étalaient entre 250 et 277 jours avec une moyenne calculée pour l'ensemble de 265,7 jours $\pm 4,6$. Cette moyenne est significativement inférieure aux durées de gestation moyennes observées en race Frisonne Pie Noire et dérivées, qui sont comprises entre 278 et 282 jours (SIGNorfit et al., I956).

\section{II. - DONnÉEs MORPhOLOGIQUES}

\section{A. - Lésions observées par les enquêteurs du C. I. A.}

Neuf fois il n'est fait mention que de la mise bas d'un "amputé » inviable. Seize fiches, par contre, comportent des suppléments d'information sur la forme de la tête, des membres ou du tronc.

Au niveau des membres les enquêteurs notent cinq fois " absence totale de membres " (ectromélie totale des quatre membres) ; une fois " absence de membres antérieur avec réduction des postérieurs à des moignons " (ectromélie totale thoracique et hémimélie pelvienne); trois fois les quatre membres se présentent sous l'aspect de " moignons terminés par des rudiments d'onglons" (hémimélie quadruple) ; une fois sous l'aspect de " moignons à l'avant " (ectromélie thoracique) et de "membres presque complets en arrière » (ectromélie distale pelvienne) ; une fois enfin, sont signalés 
"quatre membres courts terminés par des rudiments d'onglons " (ectromélie atrophique).

Pour la tête, les expressions suivantes ont été utilisées : neuf fois " tête de phoque "; une fois "tête de lièvre "; une fois tête en "museau de cochon "; trois fois tête " en forme de trompe ". Toutes se rapportent manifestement à des variétés d'otocéphalie dont le degré est malheureusement difficile à préciser.

Un enquêteur signale une fois " rein cassé "; expression qu'il faut vraisemblablement rapporter aux lésions vertébrales relevées sur l'animal examiné à Alfort.

\section{B. - Étude spéciale de l'un des monstres}

Un monstre femelle fut disséqué en novembre I96I à 1'École nationale vétérinaire d'Alfort. Les lésions suivantes ont été relevées :

$\left.\mathrm{I}^{0}\right)$ Conformation externe. Ellle est caractérisée par trois groupes de malformations siégeant au niveau des membres, de la tête et du tronc (fig. I).

a) Membres. - La partie libre des membres antérieurs fait complètement défaut (ectromélie totale). Les membres postérieurs sont considérablement réduits (hémimélie); le gauche se présente sous la forme d'un court moignon mobile de $5 \mathrm{~cm}$ environ dont l'axe squelettique est de consistance cartilagineuse ; le droit, en un moignon d'une dizaine de centimètres, orienté en haut et en dehors; il laisse reconnaître à la palpation deux éléments squelettiques : l'un proximal et fixe, l'autre distal et mobile.

b) Tête. - Les malformations céphaliques présentent le caractère commun de résulter de troubles du développement des bourgeons de la face (fig. 2).

- L'arrêt du développement des bourgeons maxillaires inférieurs se traduit par une brachygnatie accusée accompagnée des lésions consécutives classiques : atrophie de la langue qui se trouve réduite à son bourgeon pharyngien; atrésie des choanes ; tendance au rapprochement et à la fusion des oreilles sous la face, traduite, ici, par leur position en dessous et en arrière des commissures labiales; les yeux se trouvent eux-mêmes reportés vers le bas, au niveau de ces commissures. Cette série de malformations correspond à celles d'un monstre otocéphale du genre sphénocéphale (GEOHFroy SAIN'T-HIr,AIRE, I832-1836).

- La soudure incomplète des bourgeons naseaux internes se traduit par un bec de lièvre médian.

- La soudure incomplète des lames palatines par une fissure palatine.

c) Tronc. - Les malformations du tronc sont constituées par des déviations complexes de l'axe rachidien, compliquées d'ankylose. Elles donnent à la moitié postérieure du corps de l'animal une orientation spiralée. Nous pouvons décomposer de la façon suivante ces lésions rachidiennes :

a) lordose cervico-dorsale,

ß) cyphose lombo-sacrée combinée à une scoliose à concavité gauche.

$\left.2^{\circ}\right)$ A l'ouverture du cadavre, les seules malformations observées se rapportent principalement aux os des membres, des ceintures et du rachis, secondairement aux muscles, aux vaisseaux et aux nerfs correspondants (fig. 3 et 4 ).

a) Au membre antérieur droit : le scapulum est réduit à sa seule portion élargie. 

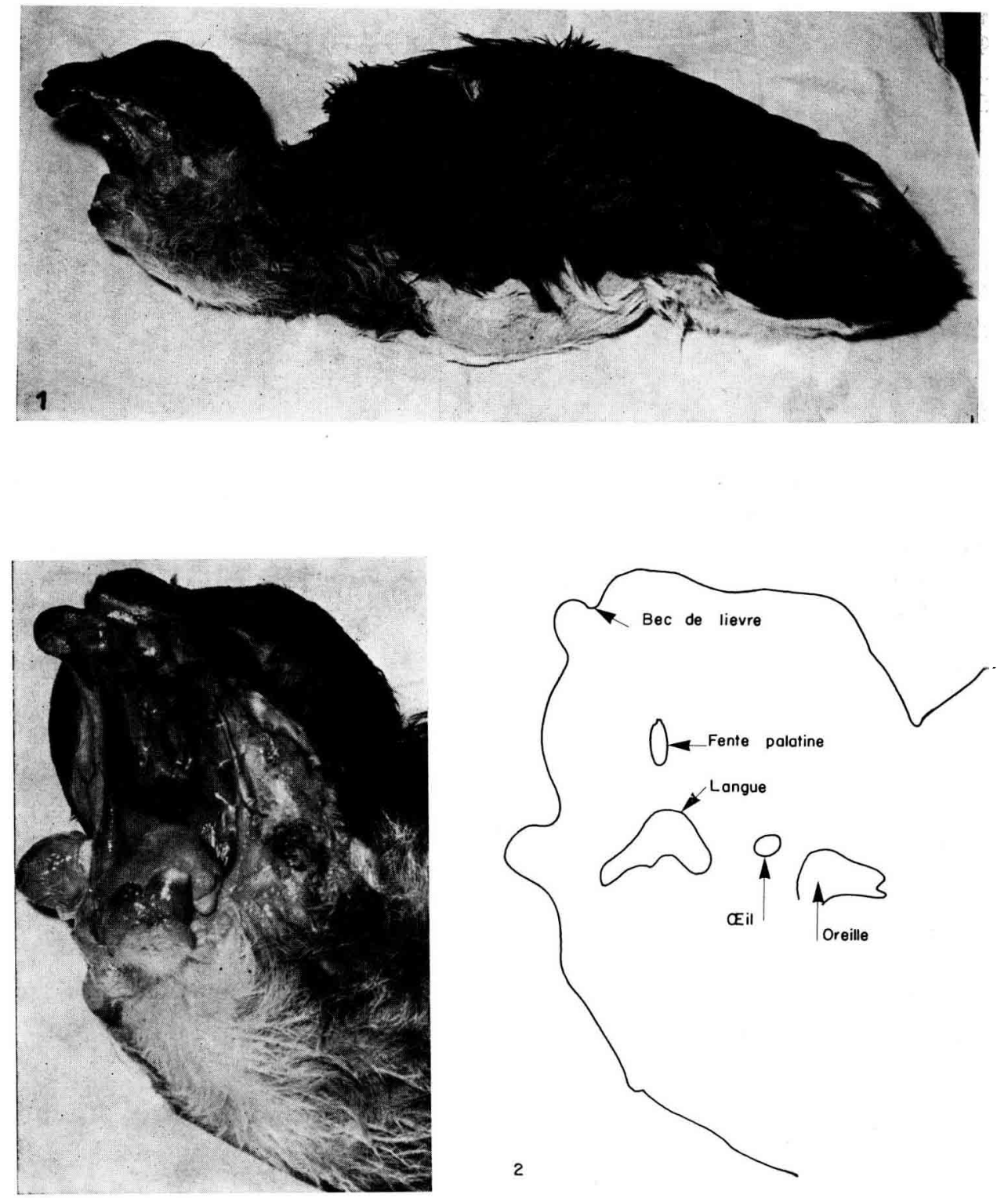

P'LANCHE I

Veau ectromèle femelle de race P'ie Noire examiné au Laboratoire d'Anatomie de l'École d'Alfort.

Fig. I. - Aspect général

Fig. 2. - Vue de la tête 
Son col et son angle inférieur font défaut. Il est dépourvu d'épine acromienne. Son angle cervical est refoulé en dedans. Il présente à son extrémité inférieure deux lames osseuses orientées dans le plan vertical et dirigées, l'antérieure en bas et en avant, la postérieure en arrière et légèrement en dedans (fig. 3).

b) Au membre antérieur gauche : le scapulum est complet, concave par sa face interne, convexe par sa face externe. Il est dépourvu d'épine acromienne et d'apophyse coracoïde. Il présente au I/3 moyen de son bord postérieur une lame osseuse orientée en arrière et en dedans, comparable à la lame osseuse correspondante du scapulum droit.

L'humérus gauche n'est représenté que par son extrémité supérieure; celle-ci

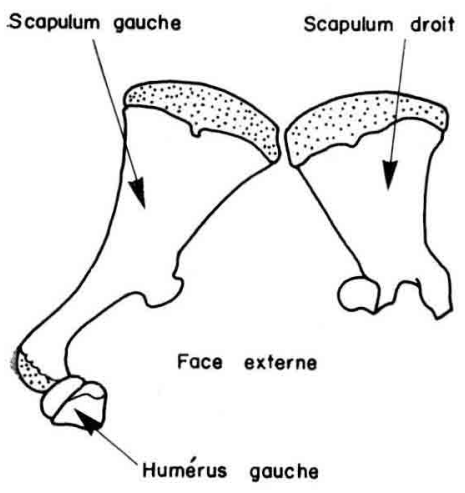

3

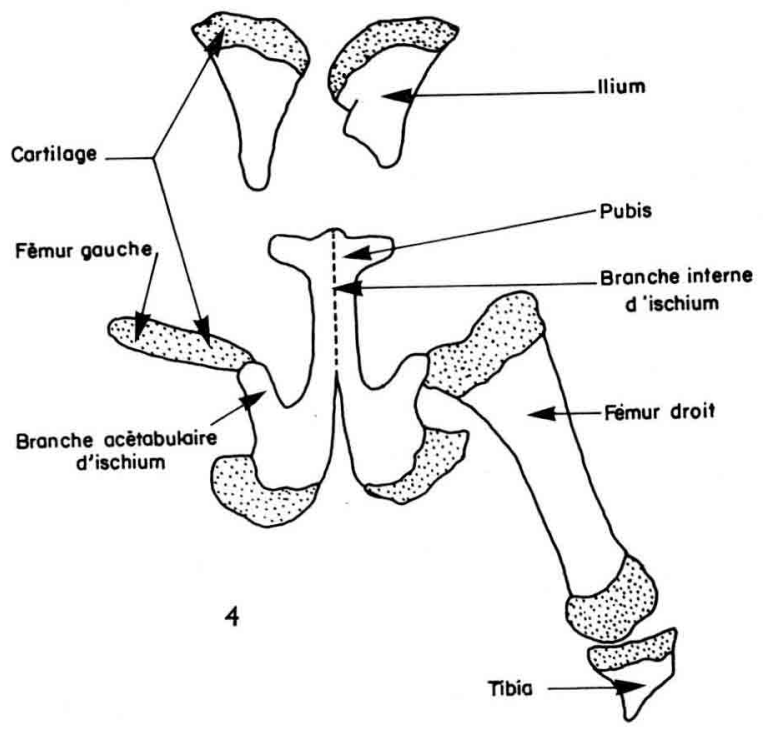

PLANCHE II

Squelette des menbres du veau monstre

FIG. 3. - Ceinture et membres thoraciques

FIG. 4. - Ceinture et membres peloiens

composée d'une surface articulaire et d'un trochin, semble correspondre au noyau interne de l'extrémité proximale de l'humérus (fig. 3).

c) La ceinture pelvienne est très incomplètement développée. Les palettes iliales sont normalement constituées mais ne possèdent pas de col. Elles sont indépendantes du reste des coxaux et ne s'articulent qu'avec le sacrum (fig. 4).

Les ischiums présentent chacun une tubérosité bien développée. I,eur branche antéro-interne paraît normale et s'unit en avant au pubis. Leur branche antéroexterne ou acétabulaire est courte, oblique en avant et en dehors et s'articule distalement avec un rudiment de fémur.

Le pubis a l'aspect d'une lame transversale fixée comme la barre d'un $T$ à l'extrémité des branches ischiales antéro-internes.

d) Le fémur gauche, rudimentaire, cartilagineux est solidement fixé par ankylose à l'extrémité de la lame acétabulaire de l'ischium gauche. Il représente à lui seul le squelette du membre (fig. 4). 
e) Le membre postérieur droit est constitué par un fémur fixé à l'extrémité de la branche acétabulaire de 1'ischium correspondant. On peut distinguer, à son extrémité supérieure, une tête et un trochanter. Aucun relief ne caractérise son extrémité inférieure (fig. 4).

Le tibia droit est représenté par une formation ostéocartilagineuse, courte et trapue, mobile sur l'extrémité inférieure du fémur sans (qu'aucun élément articulaire puisse être identifié au point de contact.

f) Les vertèbres dans les régions déviées sont défornées par tassement réciproque et soudées les unes aux autres.

\section{Conclusion}

In comparant les caractères présentés par les 26 monstres précédemment étudiés, nous pouvons constater qu'à des degrés divers deux malformations se retrouvent constamment : l'ectromélie et l'otocíphalie. L'une et l'autre peuvent être rapportées à des troubles du développement des bourgeons enbryonnaires des membres et de l'arc mandibulaire. Des variations quantitatives expliquent les différences observées d'un monstre à l'autre dans la longueur des moignons et l'aspect de la face.

A ces deux caractères principaux sont associés la mortinatalité et le raccourcissement de la durée de restation.

\section{III. - DONXIXs SUR IA PARENTÉ DES ANORMAUX}

'Tous les renseignements existant sur l'identité des pères et cles grands-pères maternels des 26 anormaux de l'enquête sont contenus dans le tableau $I$.

TABLEAU I

Renseignements ginelogiques sur les swjets anormaux

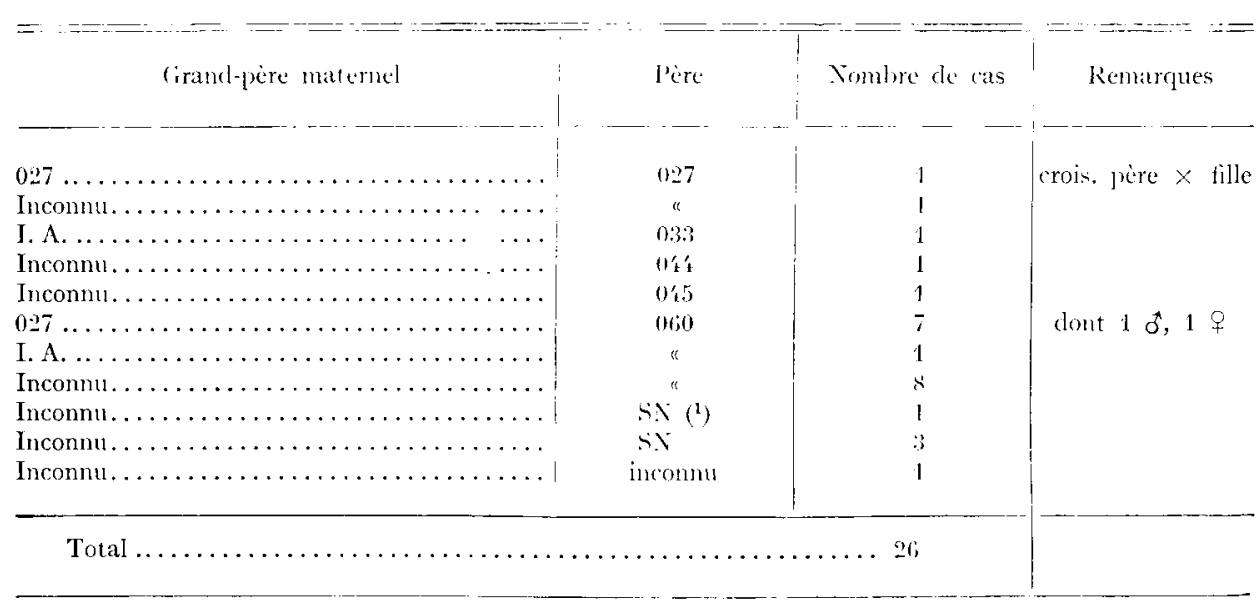

I. A. : Taureau FFPX non précisé provenant du Centre d'Insémination.

SN : Saillie naturelle.

(1) Le pire était issu d'insémination artificielle. 
On voit que les pères et les grands-pères, lorsque leur identité est connue sont tous des taureaux Frisons du Centre, dont les noms et l'origine sont précisés dans le tableau 2.

TABI,EAU 2

Identité des taureaux FFPN du C.I. A. ayant donné des reeaux "amputés"

\begin{tabular}{|c|c|c|c|c|}
\hline Xo $\therefore .1 . \Lambda$ & Koms & To ILBFFPX & No FRS & $\begin{array}{l}\text { Date de } \\
\text { naissance }\end{array}$ \\
\hline $0 \geq 7 \ldots \ldots \ldots$ & Jauste's Piet licluard ............... & 22.51 & 33562 & $23-5-51$ \\
\hline (1):3: $\ldots \ldots \ldots$ & Wytsturt Antaa's Adema $5 . . . . . . . \ldots$. & 22576 & $4 \div 697$ & $1-3 \cdot 533$ \\
\hline $015 \ldots .$. & 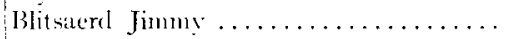 & 111 & 15950 & $12-12-5 y^{\prime}$ \\
\hline 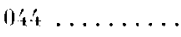 & 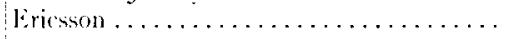 & $\because 11:$ & 4079 & $20-2-5 ;$ \\
\hline 06,0 & Jelstmer Athleet Roland... & 25273 & 672.5 & $17-11-5.1$ \\
\hline
\end{tabular}

IIPliFP : Iferd-Book de la race francaise Frisonne Pic Noire, (ambrai (Nord).

FRS: Friesth Rundee-Stambock, I,eenwarden (Frise, Pays-Ras).

Il existe entre ces taureaux des relations de parenté qui sont présentées dans la figure 5 .

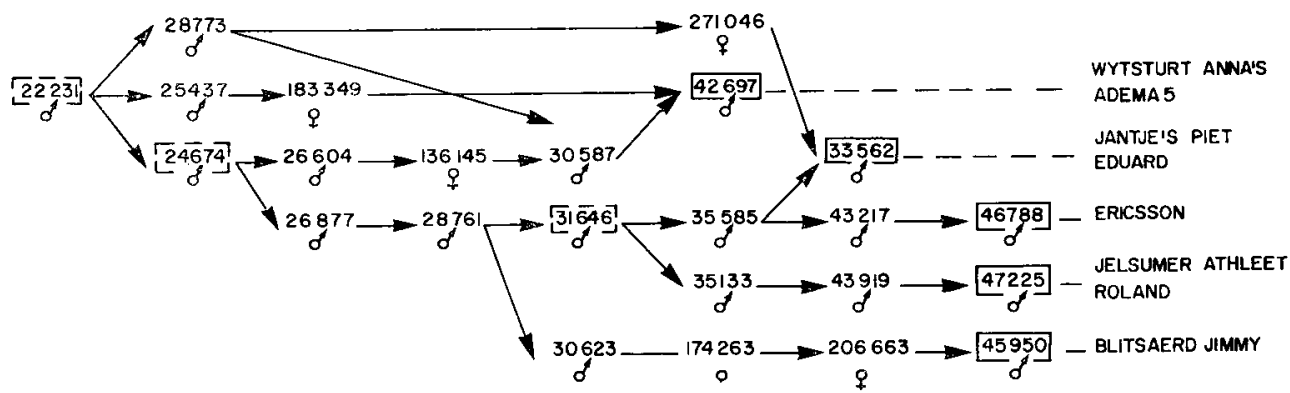

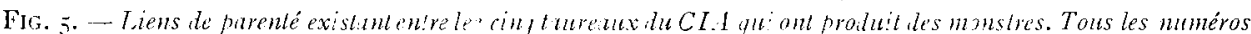
sont des munéros liRS.

$2+674:$ BANTJE'S ADEM est l'ancêtre commun le plus proche qui permet d'expliquer la présence du grène récessif chez les cinq taureaux à la fois.

22231 : AuEMA r97 peut être également pris conme ancêtre conmun, mais son rôle comme transmetteur du gine récessif défavorable semble peu probable car, alors, il y aurait cu beaucoup plus d’amputés.

36+6 : Rutje's Liduard II : des a amputés" ont été signalés, aux lays-Bas, dans sa descendance.

I,es mères des anormaux sont des vaches Française Frisonne Pie Noire (inscrites ou non) de la zone du Centre. Beaucoup sont nées d'insémination mais on ne connaît avec certitude leur père que dans un petit nombre de cas (tableau I).

Le tableau I montre :

a) que 207 (JANTJE's PIET İDUARD No FRS 33562 ) a produit des anormaux, en particulier après croisement avec une de ses filles.

b) que o6o (Jelsumer At'hleEt Roland No FRS 47225 ) croisé à des filles de 027 a produit des anormaux des deux sexes.

Dela figure 5 il ressort que 027 (No FRS 33 562) est le neveu de o6o (No FRS 47225 ). 
L'explication la plus simple pour rendre compte de ces faits est que 027 et 060 sont hétérozygotes pour un facteur récessif autosomal responsable de l'anomalie.

De même 033 (No FRS 42 697), 045 (No FRS 45950) et 044 (No FRS 46 788 ) seraient aussi hétérozygotes. Leur proche parenté avec 027 et 060 rend plausible cette hypothèse (cf. fig. 5). Toutefois comme ces trois taureaux ne sont signalés chacun que par un seul cas d'ectromélie dans leut descendance, on ne saurait conclure définitivement quant à leur génotype ; les erreurs de paternité en insémination artificielle pouvant dépasser Io $\mathrm{p}$. Ioo : vaches pleines réinséminées, erreurs de transcription...

L'anomalie que nous étudions présente donc vraisemblablement une hérédité monofactorielle de type autosomal récessif dont la pénétrance ne peut être précisée.

Tous les taureaux suspects d'hétérozygotie sont nés en Frise et inscrits au HerdBook Frison (FRS).

\section{REVUE DE LA LIT'TÉRATURE}

\section{Aspect tératorogrgue}

A. - Chez d'autres mammifères

De nombreux cas d'ectromélie ou d'otocéphalie sont signalés chez les animaux domestiques et chez l'homme. Les travaux relatifs à l'association des deux caractères sont plus rares.

Miss Lyon (1963) signale, parmi les souris de son élevage, une ectromélie totale des 4 membres, accompagnée de micrognathie. Les animaux atteints meurent avant la naissance.

JoHnson, en I940, observe chez le porc I 5 cas d'ectromélie totale des 4 membres, dont certains sont compliqués de fissure palatine et d'atrésie de l'anus.

HaNharT (I950) trouve chez l'homme une ectromélie distale (péromélie) associée à la micrognathie (forme la moins sévère de l'otocéphalie).

\section{B. - Chez les bovins}

L'ectromélie a été étudiée par DuzGunes et TuNCAY (I962) en race Brune des Alpes et par WRIEDT et MOHR (I928), GoTINK (I954) Go'IINT et al. (I955), SCHINDIER (I956), Dyrendair, et Haligren (I956) et Fischer (I959) en race frisonne Pie Noire.

En 'Turquie Duzgunes et Tuncay (1962) ont examiné 3 veaux atteints d'ectromélie simple, 2 des veaux étaient mort-nés mais le troisième a pu vivre normalement.

WRIED'T et MOHR (192S) décrivent un veau mâle de race Frisonne Pie Noire de Suède, qui présente de grandes analogies avec nos monstres, et dont ils classent ainsi les lésions : atrophiae mandibulae et maxillae (perognathia); palatum fissum; peromelia anterior (abrachia) ; peromelia posterior (adactylia); hydrocephalus. Collectant des cas identiques ils proposent le terme d'acroteriasis congenita, pour désigner l'association des anomalies de la tête et des membres.

DYRENDAHI et HALI,GREN (I956) ont retrouvé en race Frisonne Pie Noire de Suède 3 cas d'une anomalie tout à fait semblable à celle décrite par WRIEDT et MOHR 
(I928) : ectromélie et otocéphalie. Dans un cas ces auteurs ont observé une déviation de la queue. Les trois veaux étaient venus à terme, vivants.

Gotink (I954), Gotrnk et al. (I955) décrirent aux Pays-Bas des anormaux semblables à ceux de WRIEDT et MoHr. Ces veaux prématurés et mort-nés ont le corps plus ou moins couvert de longs poils. I,es appellations locales sont "veau loutre", " veau phoque ", "veau amputé ". Ils n'apparaissent qu'en Pie Noire.

SCHINDIER (I956) en race Frisonne Pie Noire d'Israël, décrit trois veaux présentant aussi de l'ectromélie, de l'otocéphalie et de l'hydrocéphalie; deux d'entre eux étaient mort-nés avant terme.

Fischer (I959), en Indonésie, observe un veau Frison Pie Noire atteint de micrognathie, fente palatine, ectromélie et hypertrychose. Il s'agit d'un foetus prélevé sur une vache morte 3 semaines avant son terme.

Ces observations jointes à la nôtre mettent en évidence la troublante fréquence de la monstruosité en race Frisonne Pie Noire.

\section{ASPEC'I HÉRÉDT'TAIRE}

\section{A. - Chez d'autres mammifères}

Io) Ectromélie simple.

Chez l'homme, les nombreux cas d'ectromélie héréditaire résumés par TouRAINE (I955) sont dus, selon les auteurs, à des mutations dominantes ou récessives.

MAUDERER (I939), chez le cheval, montre qu'une ectromélie thoracique bilatérale apparue dans l'élevage allemand de chevaux à sang froid était due à un facteur récessif.

Chez le chien, Geoffroy Sain't-Hilairl (I832-36) signale une ectromélie dont le caractère de répétition évoquait, déjà à l'époque, un mode de transmission héréditaire. Le cas étudié par LISI (IgO9) semble correspondre à une hérédité récessive.

HöHN (I942) a montré que les pedigrees des 25 moutons adactyles qu'il observe remontent à un même ancêtre et, dans douze cas, par les deux parents. Il conclut à la monofactorialité récessive de 1'anomalie et fait un rapprochement avec la même anomalie apparue en Hollande et décrite par KROOx et PLANK (I932). Dans les deux cas le gène défavorable aurait été introduit par des béliers venus de France avant I9I4.

\section{0) Ectromélie et otocéphalie.}

Pour autant qu'on sache l'anomalie observée par Miss Lyox chez la souris est transmise héréditairement sur le mode monofactoriel recessif autosomal à pénétrance complète.

JoHnson (I940) a constaté que les filles d'un verrat A croisées a un verrat B donnaient 25 amputés sur 232 naissances, rapport fort proche de $r / 8$ attendu si $A$ et $B$ étaient tous les deux hétérozygotes pour l'amputation récessive. Or, A et B sont très proches parents. Johnson conclut à l'action d'un allèle récessif de l'allèle normal.

Chez l'homme Hanhar'T (I950) n'a pu préciser si l'anomalie était héréditaire ou non. 
Io) Ectromélie simple.

In race Brune des Alpes deux des anormaux observés par I)UzGUNes et Tuxcay (r962) étaient issus d'un croisement père $\times$ fille, le troisième d'un croisement grandpère $\times$ petite-fille, le taureau étant le même dans les trois cas. I,es auteurs pensent que l'anomalie est récessive.

\section{$\left.2^{\circ}\right)$ Ectromélie et otocéphalie.}

lin race Frisonne Pie Noire de Suède, Wrient et Mohr (I928) ont trouvé que tous les parents des anormaux avaient comme ancêtre commun le fameux taureau Gallus M. 77 né en Frise orientale en I 890 introduit en Suède. Gallus eut 44 fils, $25^{\circ}$ petits-fils et 3 I9 arrière petits-fils qui furent utilisés comme reproducteurs dans le troupeau Frison Pie Noire local. I.es anormaux ont commencée à apparaître lorsque 1'on a pratiqué de la consanguinité avec les descendants de Gallus, vers I920.

Rassemblant les données de croisements père $\times$ fille oì le père était suspect d'hétérozygotie les auteurs trouvent I3 amputés pour II5 naissances. Cette proportion, très proche du rapport théorique de $1 / 8$ dans le cas de monofactorialité récessive, les conduit à adopter cette conclusion.

Les trois anormaux de DyRENDAHI, et HAI I,GREN (I956) avaient le même père : $8_{5}$ qui avait lui-même pour grand-père I06 $\mathrm{K}$. Or, I06 $\mathrm{K}$ est père d'une mère d'un anormal et grand-père des mères des deux autres. Comme dans le pedigree de Io6 $\mathrm{K}$ le taureau Gallus figure 9 fois en $8^{\text {e }}$ et $9^{\mathrm{e}}$ générations, les deux auteurs concluent qu'il s'agit d'une réapparition de l'anomalie étudiée par WRIEDT et MoHR, toujours sur le mode récessif.

Deux des 3 anormaux de SchiNDi,ER (I956) en race Frisonne d'Israël résultent de croisement père $x$ fille avec le même taureau. Les renseignements généalogiques manquent pour le troisième; Schindr,ER conclut à un facteur récessif.

Ni Gotink et al. (I955) en race Frisonne des Pays-Bas, ni Fischer (I959) en race Frisonne d'Indonésie, ne fournissent de données sur l'ascendance des anormaux.

\section{DISCUSSION}

L'anomalie française présente donc de très grandes analogies avec celle que Wriedt et IoHr, DYRENDAHL et HAI, Grex, Gotink et al., SchiNdi,ER et Fischer ont décrites :

a) l'aspect tératologique est le même : ectromélie, otocéphalie, mortinatalité et mise bas avant terme.

b) le type génétique monofactoriel, autosonal et récessif semble être identique à ceux que WRIEDT et MoHr en Suède, et SchIxDLER en Israël mirent en éridence et que DYRENDAHI, et HALLGREN ont confirmés.

c) dans tous les cas il s'agit de la race Frisonne Pie Noire.

Comme Gallus les cinq taureaux français suspects sont nés en Frise et inscrits au Herd-Book Frison (FRS). Les communications de GoTink et Gotrink et al., prouvent la latence de l'anomalie en race Frisonne Pie Noire des Pays-Bas. Mieux, 
trois taureaux du Centre ont pour ascendant conmun RUTJE's FintaR1) II (No FRS 3I 646) (cf. fig. 5) connu en Hollande pour avoir produit des " amputés", Politifk

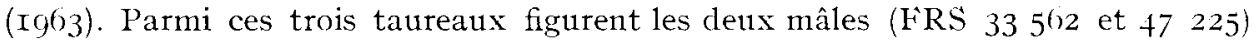
dont l'hétérozygotie est attestée par plus d'un descendant ectromèle.

Nous regrettons que Schrndren et Fischer n'aient pas donné les identités de leurs taureaux, et, d'autre part, qu'il ait été impossible de rechercher les liens de parenté éventuels entre Gallus et les taureaux suspects d'hétérozygotie. Il n'en reste pas moins que comme celle de WRIEDT et MOHR en Suède et, probablement, comme celles de Schind,ER en Israël et Fischer en Indonésie, l'anomalie française semble provenir de Frise.

La récessivité apparaissait complète d'après les travaux de WRIEDT et MoHR (Ig28), nos données ne nous permettent pas de préciser ce point.

\title{
CONCIUSION
}

I a monstruosité que nous décrivons, apparue en race Française Frisonne Pie Noire, se traduit tératologiquement par l'ectromélie, l'otocéphalie, la mortinatalité et la mise bas avant terme. Génétiquement, elle peut être rapportée à une hérédité monofactorielle, autosomale et récessive.

Ces caractères la rapprochent d'anomalies semblables observées parmi la descendance de géniteurs de race Frisonne Pie Noire dans divers pays.

Resu pour publication en juillet 1963.

\section{SUMMARY}

\author{
HERLDITARY ECTROMELIA AND OTOCEPHALIA IN « FRANÇAISE FRISONNE PIE NOIRE » \\ BREED OF CATTLE
}

An anomaly has repeatedly appeared in the « Française Frisonne Pie Noire » breed of cattle within the zone of an Insemination Centre in the North of France.

In all the affected cases, more or less complete absence of limbs (ectromelia) is accompanied by malformations of the head (otocephalia). The abnormal animals are still-born after a shorter than normal length of gestation.

The most probable genetic hypothesis, one that is accepted in published work, is that the characteristic is conditioned by an autosomal recessive factor.

As the sires of the prodigies which we have studied come from Friesland, and since they are related to a bull which, in the Netherlands, has given " amputated " calves, there is cause to think that the anomaly which has arisen is a reappearance of recessive ectromelia associated with otocephalia, latent in the Black Pied Friesian breed: the "acroteriasis congenita " of WRIEDT and MOHR.

\section{RÉFÉRENCES BIBLIOGRAPHIQUES}

Dezgunes O., Texcay A., ig62. The amputated condition in brown swiss cattle. J. Hered., 53, 226. Dyrendail S., Hallgren N., 1956. Nouveau cas d'acroteriasis congenita en race des plaines (en suédois). Nord. Vet. Med., 8, $959-65$. 
Fischer H., 1959. Acroteriasis congenita in a Holstein - Friesian calf fotus. Hemera zoa, 66, 9I-2.

Geoffroy Simt Hilatre I., 1832-36. Histoire générale et particulière des anomalies de lorganisation ches l'homme el chez les animaux, tome 2, 206-237, 420-437, J. B. Baillère, Paris.

Comsk IT. M., Stérilité chez les bovins. II. Avortement de l'embryon (en néerlandais). Keur-Stamboker, 2, 223-24

Gotrnk W. MI., De Groot Th., Stegenga Th., 1955. Défauts héréditaires des bovins (en néerlandais). Landbousk. Tijdschr., 67, 629-72.

Hanhar' l.. i 950. Über die Kombination von Peromelie mit Mikrognathie, ein neues Syndrom beim Menschen, entsprechend der Akroteriasis congenita von Wk1edt und IOHR beim Rinde. drch. Klans. Silft. Vererbforsch., 25, $533^{-4}+3$.

I0̈Hx W., I942. Adaktylie, ein neuer rezessiver Letalfaktor in der Schafzucht. Disch. Tierärsll. Wschr., 50, 349-50.

Jornsox L. L., 1940. "Streamlined " pigs. A new legless mutation. J. Mered., 31, 239-42.

Kobozimfr N., Pomriaskinsky-Kobozievf X. A., i943. Précis de Génétique appliquée à la médecine véterinaine, 91, Vigot Frères, Paris.

Kroon H. M., Plank G. M. v. d., т932. Einige subletale Falitoren bei IIaustieren in den Niederlanden. Biol. gen., 8, 2 I $3^{-2} \mathrm{r} 8$.

Lisı V., I909. Monstruosité observée héréditairement chez quelques petits chiens. Rev. vét., 34, 432.

Irox (Miss Mary F.) |MRC radiobiological Research Unit, I.uRwer., Berks, Lngland|. Iy63. Communication personnclle.

Macderer 1I., 1939. Abrachie und Torticollis, remessive Letalfaktoren in der l'ferdzucht. Diss., Hannover.

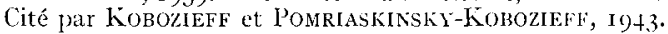

Politiki R. D. [Land souwhogeschool Wagknisgks, Vederland]. rg63. Communication personnelle.

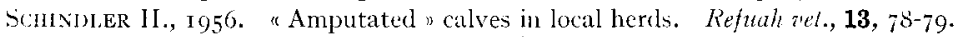

Sigxoret J. P., Poly J., Vissac 13., I956. Étude statistique des causes de variation de quelques paramètres du cycle de reproduction des vaches laitieres. I. La durée de gestation dans les races lovines Normande et l'rançaise Frisonne Pie Noire. Ann. Zootech., 6, 273-204.

Tulraine A., 1955. L'hérédité en médecine, 673-675, Masson et Cie, Paris.

WriedT C., MoHR O. L., ig2s. Amputated, a recessive lethal in cattle. 1. Genet., 20, i87-215. 